\title{
artigo
}

Xavier, O.E.; Valente Filho, C.C.; Dias, J.H.A.; Rêgo, R.S.; Dutra da Silva, R.C.; Guerra, H.S.;

Perfil dos pacientes atendidos em um ambulatório universitário de especialidades

\section{Perfil dos pacientes atendidos em um ambulatório universitário de especialidades}

\author{
Profile of patients seen at a university specialized outpatient clinic \\ Perfil de los pacientes atendidos en una consulta externa especializada universitaria
}

\begin{abstract}
RESUMO
Objetivo: Caracterizar o perfil dos pacientes atendidos no ambulatório de especialidades médicas de uma faculdade de Medicina de um município goiano. Métodos: Estudo documental e retrospectivo, analisando as variáveis relacionadas ao perfil do usuário. Os dados foram apresentados como frequência absoluta (n) e relativa (\%) e idade como média e desvio-padrão (DP). Resultados: A maioria dos usuários eram do sexo masculino (58,6\%), idade média de 42 anos, solteira $(50,6 \%)$, economicamente ativa $(54,4 \%)$ e residente em Aparecida de Goiânia $(96,4 \%)$. Predominaram os sedentários $(65,2 \%)$, não etilistas $(66,7 \%)$ e não tabagistas $(66,6 \%)$, eutróficos $(59,3 \%)$ e que utilizavam de uma a cinco medicações $(66,1 \%)$. As especialidades com maior frequência de consultas foram a Pneumologia, Pediatria e Dermatologia. Muitos prontuários faltavam informações importantes para compreensão do perfil. Conclusão: A análise dessas informações possibilita melhor capacitar os profissionais e acadêmicos para uma triagem e intervenções mais eficientes.
\end{abstract}

DESCRITORES: Assistência ambulatorial; Registros médicos; Perfil de saúde.

\section{ABSTRACT}

Objective: To characterize the profile of patients seen at the medical specialties outpatient clinic of a Faculty of Medicine in a city in Goiás. Methods: Documentary and retrospective study, analyzing the variables related to the user profile. Data were presented as absolute ( $\mathrm{n})$ and relative (\%) frequency and age as mean and standard deviation (SD). Results: Most users were male $(58.6 \%)$, mean age 42 years, single (50.6\%), economically active (54.4\%) and resident in Aparecida de Goiânia (96.4\%). Sedentary people $(65.2 \%)$, non-drinkers $(66.7 \%)$ and non-smokers $(66.6 \%)$, eutrophic $(59.3 \%)$ and who used from one to five medications (66.1\%) predominated. The specialties with the highest frequency of consultations were Pneumology, Pediatrics and Dermatology. Many medical records lacked important information for understanding the profile. Conclusion: The analysis of this information makes it possible to better train professionals and academics for more efficient screening and interventions.

DESCRIPTORS: Ambulatory care; Medical records; Health profile.

\section{RESUMEN}

Objetivo: Caracterizar el perfil de los pacientes atendidos en el ambulatorio de especialidades médicas de una Facultad de Medicina de una ciudad de Goiás. Métodos: Estudio documental y retrospectivo, analizando las variables relacionadas con el perfil del usuario. Los datos se presentaron como frecuencia absoluta (n) y relativa (\%) y la edad como media y desviación estándar (DE). Resultados: la mayoría de los usuarios eran hombres (58,6\%), edad media 42 años, solteros (50,6\%), económicamente activos $(54,4 \%)$ y residentes en Aparecida de Goiânia $(96,4 \%)$. Predominaron las personas sedentarias $(65,2 \%)$, no bebedores $(66,7 \%)$ y no fumadores $(66,6 \%)$, eutróficos $(59,3 \%)$ y que consumían de uno a cinco medicamentos $(66,1 \%)$. Las especialidades con mayor frecuencia de consultas fueron Neumología, Pediatría y Dermatología. Muchos registros médicos carecían de información importante para comprender el perfil. Conclusión: El análisis de esta información permite capacitar mejor a los profesionales y académicos para un cribado e intervenciones más eficientes.

DESCRIPTORES: Atención ambulatoria; Registros médicos; Perfil de salud.

RECEBIDO EM: 01/06/2021 APROVADO EM: 07/06/2021

\section{Oemis Eduardo Xavier}

Acadêmico de Medicina da Faculdade de Medicina, UniRV Campus Aparecida, Aparecida de Goiânia, Goiás, Brasil.

ORCID: 0000-0001-9852-5312 


\section{Carlos Chaves Valente Filho}

Acadêmico de Medicina da Faculdade de Medicina, UniRV Campus Aparecida, Aparecida de Goiânia, Goiás, Brasil. ORCID: 0000-0002-4337-2264

\section{Jorge Henrique Assunção Dias}

Acadêmico de Medicina da Faculdade de Medicina, UniRV Campus Aparecida, Aparecida de Goiânia, Goiás, Brasil. ORCID: 0000-0001-6446-2552

\section{Ruth da Silva Rêgo}

Acadêmica de Medicina da Faculdade de Medicina, UniRV Campus Aparecida, Aparecida de Goiânia, Goiás, Brasil. ORCID: 0000-0002-4729-6465

\section{Renato Canevari Dutra da Silva}

Fisioterapeuta, Mestre em Ciências da Saúde (UNB), Doutorando em Saúde Coletiva (UNISINOS), Professor Adjunto da Faculdade de Fisioterapia da Universidade de Rio Verde (UniRV), Campus Rio Verde, Goiás, Brasil.

ORCID: 0000-0002-6428-2823

\section{Heloísa Silva Guerra}

Fisioterapeuta, Mestre em Saúde Coletiva (UFG), Doutoranda em Saúde Coletiva (UNISINOS), Professora Adjunto da Faculdade de Medicina da Universidade de Rio Verde (UniRV), Campus Aparecida, Aparecida de Goiânia, Goiás, Brasil.

ORCID: 0000-0002-0617-8112

\section{INTRODUÇÃO}

0 Sistema Único de Saúde (SUS) tem como princípios básicos a universalidade, equidade e a integralidade da assistência. A integralidade pode ser entendida como um conjunto articulado e contínuo das ações e serviços preventivos e curativos, individuais e coletivos, exigidos para cada caso em todos os níveis de complexidade do sistema ${ }^{1}$.

$\mathrm{Na}$ organização do SUS, conforme prevê o Decreto ${ }^{\circ} 7.508$ de 2011, o conjunto de ações e serviços de saúde articulados em níveis de complexidade crescente, com a finalidade de garantir a integralidade da assistência à saúde, é denominado de Rede de Assistência à Saúde (RAS)². Na RAS os serviços estão distribuídos em três níveis de atenção: primário, secundário e terciário; e essa organização é uma estratégia para superação do modo fragmentado de operar a assistência e a gestão em saúde ${ }^{3}$.

A atenção secundária caracteriza-se por serviços especializados em nível ambulatorial e hospitalar, com densidade tecnológica intermediária entre a atenção primária e terciária, também conhecido como média complexidade ${ }^{3}$. Neste nível, encontram-se os serviços médicos especializados, de apoio diagnóstico e terapêutico e atendimento de urgência e emergência.
A integralidade

pode ser entendida

como um conjunto

articulado e

contínuo das

ações e serviços

preventivos e

curativos, individuais

e coletivos, exigidos

para cada caso em

todos os níveis

de complexidade

do sistema
As clínicas escolas, vinculadas à Instituições de Ensino Superior (IES), estão inseridas na RAS e no geral apresentam uma dupla função: proporcionar ao acadêmico a prática clínica dentro de sua profissão; e fazer com que a Universidade cumpra seu papel social, por meio da prestação de serviços à comunidade ${ }^{4}$.

Um ambulatório de especialidades de instituições de ensino é o ambiente ideal para os acadêmicos, pois são profissionais em formação e estão sendo preparados para seu futuro ofício acompanhando os fluxos de atendimentos e reflexões sobre a sua formação por meio da realização de consulta sob orientação de um profissional capacitado. Nesse contexto, o aluno tem a oportunidade de aplicar os conhecimentos teóricos na prática clínica, aperfeiçoando o processo de ensino e aprendizagem. Outro benefício desse serviço-escola é fornecer atendimento à população, principalmente a de baixa renda, que tem acesso mais restrito aos serviços de saúde 5 .

Soma-se à isso a aquisição de competências para julgamento moral, que valoriza aspectos éticos e humanos durante a educação médica que ocorre na atenção primária e ambulatorial. $\mathrm{O}$ contato ao longo do curso com pacientes do Sistema Único de Saúde, com participação ativa no atendimento, traz inevitavelmente para a 
discussão alguns pontos da medicina centrada no paciente ${ }^{6}$.

O método clínico centrado na pessoa (MCCP) fundamenta-se na investigação, de forma equilibrada, tanto da doença em si, quanto da experiência de estar doente, considerando o contexto de vida do indivíduo. Esse processo requer escuta qualificada e atenta da história e necessidades do paciente, no sentido de compreender e melhor conduzir cada caso ${ }^{7}$.

Todos esses aspectos tornam-se imprescindíveis na educação médica, que deve se pautar pela construção, no decorrer da graduação, de um profissional com formação generalista, humanista, crítica e reflexiva, capacitado a atuar frente ao processo saúde-doença, baseado em princípios éticos, nos diferentes níveis de atenção, visando a integralidade da assistência e o desenvolvimento do senso de responsabilidade social e compromisso com a cidadania ${ }^{8}$.

Conhecer o contexto de vida do paciente é de suma importância para compreender sua integralidade, suas condições de vida e possíveis fatores que podem explicar o processo saúde-doença. A análise da população que frequenta um serviço médico é relevante para o conhecimento geral dos pacientes, servindo como motor do atendimento adequado para esses indivíduos que buscam o serviço.

Diante do exposto, o objetivo deste estudo foi caracterizar o perfil dos pacientes atendidos no ambulatório de especialidades médicas de uma faculdade de Medicina de um município de Goiás.

\section{MÉTODOS}

Trata-se de um estudo documental, retrospectivo, com dados coletados em prontuários de pacientes atendidos no Ambulatório de Especialidades Médicas da Faculdade de Medicina (FAMED) da Universidade de Rio Verde, Campus Aparecida de Goiânia.

Aparecida de Goiânia está localizada na região metropolitana de Goiânia e possui quase 500 mil habitantes. O município conta com 52 locais de atendimentos, sendo eles divididos em: 40 unidades básicas de

\section{O método clínico}

centrado na

pessoa (MCCP)

fundamenta-se na

investigação, de

forma equilibrada,

tanto da doença

em si, quanto da

experiência de estar

doente, considerando

o contexto de vida

do indivíduo. Esse

processo requer

escuta qualificada

e atenta da história

e necessidades do

paciente, no sentido

de compreender e

\section{melhor conduzir}

cada caso saúde (UBS), 01 maternidade, 01 serviço de atendimento especializado (SAE), 01 centro clínico municipal (CCM), 01 núcleo de cuidados em saúde mental (NCSM), 01 Serviço de atendimento de emergência em saúde mental, 01 hospital municipal, 01 ambulatório municipal, 04 Centros de Atenção Psicossocial (CAPS), 02 Centros de Atenção Integrada à Saúde (CAIS) e 03 Unidades de Pronto Atendimento (UPA).

O Ambulatório de Especialidades Médicas da FAMED, agregou-se à RAS de Aparecida desde fevereiro de 2017 e realiza atendimento ambulatorial com os acadêmicos de Medicina, a partir do quarto período da graduação, nas seguintes especialidades: Cardiologia, Cirurgia Geral, Coloproctologia, Dermatologia, Endocrinologia, Endocrinologia pediátrica, Gastroenterologia, Ginecologia, Infectologia, Nefrologia, Neurologia, Pediatria, Pneumologia, Reumatologia e Urologia.

Para a operacionalização da coleta de dados da pesquisa, foi utilizado um instrumento que serviu como roteiro para a extração das informações do prontuário, necessárias à caracterização do perfil dos pacientes. A coleta aconteceu no período compreendido entre dezembro de 2019 e fevereiro de 2020 e incluiu todos os prontuários abertos na unidade desde sua abertura até 31 de dezembro de 2019. As variáveis analisadas foram: local de residência, procedência, sexo, idade, escolaridade, profissão, estado civil, renda familiar, data da primeira consulta, especialidade, número de retornos na especialidade de origem, hipótese diagnóstica, comorbidades, hábitos de vida, Índice de Massa Corporal (IMC) e número de medicamentos em uso.

Os dados foram analisados no programa STATA, versão 14.0 (StataCorp, 2015). Inicialmente, foi realizado o teste de Kolmogorov-Smirnov com correção de Lillifors para verificação da normalidade da variável idade. A seguir, foi realizada análise descritiva das variáveis. As variáveis quantitativas do perfil dos pacientes foram apresentadas como frequência absoluta (n) e relativa (\%) e idade como média e desvio-padrão (DP), mediana, intervalo interquartil (IIQ), mínimo e máximo. 
Tabela 1. Análise descritiva das variáveis relacionadas ao perfil sociodemográfico dos pacientes atendidos em um ambulatório universitário de especialidades. Aparecida de Goiânia - G0, 2020 ( $n=1.684)$

\section{VARIÁVEIS}

\section{Sexo}

Feminino

Masculino

SI: 2

Faixa etária (anos)

0-9

10-19

225

189

20-39

338

40-59

422

$>60$

510

Escolaridade

Não estudou/ensino fundamental incompleto

Ensino fundamental completo

Ensino médio incompleto

Ensino médio completo

Ensino superior incompleto

Ensino superior completo

SI: 1.627

\section{Ocupação}

Emprego

Estudante

Aposentado

192

Desempregado

SI/NSA: 534

\section{Estado civil}

Solteiro

Casado

Viúvo

Divorciado

SI/NSA: 563

\section{Cidade de residência}

Aparecida de Goiânia

Goiânia

Outras cidades

SI: 34

Unidade da federação de procedência

Goiás

Outro estado
$\%$

41,4

58,6

10,5

6,0

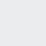

$\begin{array}{lcc}\text { Estado civil } & 567 & 50,6 \\ \text { Solteiro } & 406 & 36,2 \\ \text { Casado } & 81 & 7,2 \\ \text { Viúvo } & 67 & 6,0 \\ \text { Divorciado } & & \\ \text { SI/NSA: 563 } & & 96,4 \\ \text { Cidade de residência } & 1.591 & 2,2 \\ \text { Aparecida de Goiânia } & 36 & 1,4 \\ \text { Goiânia } & 23 & \\ \text { Outras cidades } & & 59,7 \\ \text { SI: } 34 & & 40,3 \\ \text { Unidade da federação de procedência } & 770 & \\ \text { Goiás } & 519 & \\ \text { Outro estado } & & \end{array}$




\section{artigo}

Xavier, O.E.; Valente Filho, C.C.; Dias, J.H.A.; Rêgo, R.S.; Dutra da Silva, R.C.; Guerra, H.S.;

Perfil dos pacientes atendidos em um ambulatório universitário de especialidades
SI: 395
Média (DP)
Mediana (IIQ)
Idade (anos)
$42,2(24,7)$
$45(20-63)$
Abreviações: DP = Desvio-padrão; SI= Sem informação; SI/NSA=Não se aplica. IIQ=Intervalo Interquartil.

Tabela 2. Análise descritiva das variáveis relacionadas ao atendimento em um ambulatório universitário de especialidades. Aparecida de Goiânia - GO, 2020 ( $n=1.684)$.

VARIÁVEIS

Ano da primeira consulta

2017

2018

2019

SI: 232

\section{Especialidade de origem}

Cirurgia geral

27

242

Dermatologia

Endocrinopediatria

52

15

133

161

108

26

244

278

14,6

Gastroenterologia

Ginecologia

Infectologia

Nefrologia

Neurologia

Pediatria

Pneumologia

Reumatologia

Urologia

Coloproctologia

Endocrinologia

Cardiologia

SI: 27

\section{Consultou em outra especialidade}

Não

1.629

97,1

Sim

48

2,9

SI: 7

Abreviações: SI/NSA= Não se aplica.

namento, evidenciando a importância da unidade para o município e demonstrando que o volume de consultas aumentou gradativamente, melhorando acesso e contribuindo para a diminuição do tempo de espera por uma consulta especializada.

A principal finalidade de se determinar o perfil de usuários de um serviço é captar informações relevantes que possam melhorar a disponibilidade destes serviços, além de colaborar para a seleção e capacitação de recursos humanos e uma melhor articulação com outros estabelecimentos de assistência à saúde9. Além disso, as consultas ambulatoriais contribuem substancialmente na formação do médico generalista uma vez que o estudante tem a oportunidade de vivenciar a integração entre os níveis de atenção do SUS e aprofundar o conhecimento em áreas específicas da medicina ${ }^{10}$.

Os resultados do presente estudo mostraram um perfil de usuários predominantemente do sexo masculino, jovem, solteira e economicamente ativa. A predominância do sexo masculino corrobora outros estudos que traçaram perfil de pacientes em atendimentos ambulatoriais ${ }^{11,12}$. No entanto, contrariam outras pesquisas que apontam predomínio do público feminino e de idosos entre aqueles que mais buscam o sistema de saúde ${ }^{13-16}$.

A média de idade encontrada concorda com outro estudo sobre pacientes atendidos num serviço de dermatologia de São Paulo ${ }^{17}$, por volta de 42 anos. Vale ressaltar que o presente estudo se refere a um ambulatório de multiespecialidades, incluindo pediatria e dermatologia, que podem ser responsáveis pela maioria do público jovem. Observou-se também um percentual grande de prontuários sem a informação de escolaridade, porém, dentre os que constavam, a maioria dos pacientes não tinha estudo ou apresentava apenas o Ensino Fundamental Incompleto, concordando com estudo semelhante onde $50,2 \%$ dos pacientes eram analfabetos ou tinham escolaridade menor que 4 anos ${ }^{18}$.

$\mathrm{O}$ estudo de Sena et $\mathrm{al}^{17}$, que descreve o perfil epidemiológico de um ambulatório de dermatologia, apresentou o mesmo resultado percentual de prontuários sem preenchimento do nível de escolaridade (97\%). Esse achado sugere que o questionamento acerca da escolaridade é bastante negligenciado nos atendimentos médicos, o que pode impactar no sucesso da consulta e adesão ao tratamento proposto. Ademais, já é conhecida a relação entre escolaridade e saúde, onde pessoas com menor escolaridade apresentam um perfil mais adverso de saúde, com maiores prevalências de algumas doenças, menor realização de consultas preventivas e maiores taxas de internação ${ }^{19}$.

Considerando o estilo de vida dos usuários, os achados refletem alguns dos maus hábitos da população brasileira que con- 
tribuem para o aparecimento e perpetuação de doenças crônicas não transmissíveis (DCNT), como a hipertensão arterial (HA) por exemplo. A maioria da amostra não praticava atividade física, o que gera grande preocupação. As evidências científicas apontam uma crescente prevalência de HA entre jovens, motivada sobretudo pela obesidade e estilo de vida sedentário. A prática de atividade física se constitui um fator protetor preventivo importantíssimo contra o desenvolvimento da HA, doenças cardiovasculares e mortalidade por todas as causas ${ }^{20}$.

Quase um terço dos participantes encontrava-se acima do peso considerado normal, embora a maior parte dos atendidos eram eutróficos e, ainda, muitos prontuários não continham a informação referente a peso e altura. No estudo sobre o perfil epidemiológico de pacientes com colelitíase atendidos em um ambulatório de cirurgia e em outro que avaliou pacientes atendidos no ambulatório de avaliação pré-anestésica, notou-se que a maior parte dos usuários apresentavam IMC acima do considerado ideal ${ }^{9,21}$. O estado nutricional do indivíduo sofre influência de diversos determinantes, a depender do ambiente, cultura, condições sociais, demográficas e epidemiológicas em que eles estão inseridos, sendo que estes determinantes impactam sua saúde e qualidade de vida ${ }^{21}$.

$\mathrm{O}$ uso de muitas medicações foi frequente na amostra estudada, sendo que $16,8 \%$ os pacientes faziam uso de 5 ou mais medicamentos. A alta taxa de polifarmácia pode estar relacionada ao número de idosos da amostra $(30,3 \%)$ e à sobreposição de doenças crônicas e infecciosas agudas nos jovens, como asma e infecção do trato urinário (ITU).

No tocante ao atendimento, a maior frequência de consultas na especialidade Pneumologia pode ser justificada pela predominância do sexo masculino no presente estudo, uma vez que as principais doenças pneumológicas estão relacionadas ao hábito de fumar. Historicamente mais comum entre os homens, esse hábito leva a inflamação crônica das vias aéreas, que acaba ocasionando alterações estruturais nos alvéolos, fibrose, dano ao epitélio mucociliar e hipersecreção brônquica. Esses fatores predispõem fortemente ao aparecimento de doenças pulmonares, além das atividades laborais relacionadas ao sexo masculino, como o trabalho nas pedreiras, presentes na região do ambulatório analisado por este estudo ${ }^{22}$.

A ocorrência de patologias respiratórias pode estar relacionada ao fato de o município de Aparecida de Goiânia possuir um dos principais Polos Industriais do Estado de Goiás, representando 9,74\% do total de indústrias do estado ${ }^{23}$.

Os poluentes aéreos emitidos por essas empresas, em contato com o epitélio respiratório, podem provocar reações adversas no organismo, com impacto principalmente no sistema cardiovascular e aparelho respiratório, tornando esse tema relevante e atual em diversas partes do mundo ${ }^{24}$. A alta prevalência no diagnóstico de dermatite atópica no presente estudo, a qual possui fisiopatologia de caráter inflamatório e pode ser exacerbada com a exposição à poluentes ambientais, também pode se referir à presença dessas empresas na região; o que corrobora para um grande fluxo de atendimento na especialidade de dermatologia.

O diagnóstico de Doença Renal Crônica (DRC), também prevalente no presente estudo, mais uma vez pode refletir a preponderância da população masculina neste serviço, principalmente pela relutância dessa população em procurar atendimento de saúde preventivamente por causa da cultura, de valores sociais, desinformação ou por considerar a doença como sinal de fragilidade ${ }^{25}$.

Essas crenças fazem com que os homens se cuidem menos, se exponham mais a situações de risco, tenham menor adesão aos tratamentos e só busquem os serviços quando em estágio mais avançado, predispondo-os a doenças graves e crônicas. Isso se relaciona à construção da masculinidade focada na formação de um ser másculo, onde não se permite a expressão de suas dores e fragilidades ${ }^{26}$.

A análise das informações sobre os usuários do nível ambulatorial no âmbito do
SUS é muito relevante, uma vez que é possível, via prontuários, melhor capacitar os profissionais para uma triagem e intervenções mais eficientes, permitindo o acesso a dados sobre o perfil epidemiológico do paciente em questão, histórias patológicas pregressas, condutas tomadas, identificação de doenças de base e exames complementares realizados. Essas informações podem ser acessadas e interpretadas de melhor forma quando estão contidas em base de dados eletrônicos, no entanto essa não é uma realidade em muitos serviços vinculados ao poder público.

Uma das limitações do presente estudo foi a escassez de informações registradas nos prontuários dos pacientes e a caligrafia extremamente confusa de alguns profissionais e acadêmicos. A incompletude dos dados revela um déficit no processo de formação profissional, onde uma anamnese completa se faz necessária.

A anamnese pode ser compreendida como um dos desafios da educação médica, que aponta a mudança de postura do entrevistador para a de ouvinte, ou seja, de indagador para a de cuidador do processo saúde-doença das pessoas, sinalizando sua importância na "arte" da escuta para identificação das necessidades de saúde e de cuidado no eixo da integralidade ${ }^{27}$. Ademais, o prontuário é um documento legal, de caráter sigiloso e científico, que serve de comunicação oficial entre os diversos profissionais da equipe multidisciplinar, permitindo a continuidade dos cuidados prestados ao indivíduo e constituindo-se como referência para questões administrativas, legais e financeiras. A falta de informações nos prontuários pode comprometer suas funções e utilidades.

\section{CONCLUSÃO}

O perfil de pacientes do ambulatório de Especialidades Médicas da FAMED evidenciou uma população de usuários em sua maioria jovens, do sexo masculino, solteira, economicamente ativa, sedentária e em uso de uma a cinco medicações. A maioria dos diagnósticos apresentados 


\section{artigo}

Xavier, O.E.; Valente Filho, C.C.; Dias, J.H.A.; Rêgo, R.S.; Dutra da Silva, R.C.; Guerra, H.S.;

Perfil dos pacientes atendidos em um ambulatório universitário de especialidades

estavam relacionados às áreas de Pneumologia, Pediatria e Dermatologia.

Anseia-se que com o perfil epidemiológico traçado no presente estudo, haja um melhor planejamento estratégico por parte da instituição de ensino, no sentido de otimizar a organização dos atendimentos, capacitar profissionais e acadêmicos quan- to à importância do registro de informações, bem como proporcionar um melhor atendimento aos usuários que utilizam esse serviço.

\section{REFERÊNCIAS}

1. Brasil. Lei n. 8.080 de 19 de setembro de 1990. Lei Orgânica da Saúde. Diário Oficial da União, Brasília, DF, p. 18.055, 20 set. 1990.

2. Brasil. Ministério da Saúde. Decreto 7.508 de 28 de junho de 2011. Regulamenta a Lei $n^{\circ} 8.080$ de 19 de setembro de 1990. Diário Oficial da União, Seção 1, 29 jun. 2011.

3. Brasil. Portaria GM/MS no 4.279, de 30 de dezembro de 2010 Estabelece diretrizes para a organização da Rede de Atenção à Saúde no âmbito do SUS. Brasilia: Ministério da Saúde; 2010.

4. Zilli MG, Santos APS, Yamaguchi CK, Borges LA. Contribuições de uma clínica escola no atendimento em saúde no Sul de Santa Catarina. RIES. 2017; 6(1):105-116.

5. Fernandes LFB, Silvares EFM, Miyazaki MCOS. Caracterização da população atendida em ambulatório de Psicologia da Saúde de um hospital-escola. Contextos Clínic. 2017;10(2):145-156.

6. Ribeiro MMF, Amaral CFS. Medicina centrada no paciente e ensino médico: a importância do cuidado com a pessoa e o poder médico. Rev. bras. educ. med. 2008; 32(1):90-97.

7. Chazan ACS, Silveira LMC, Favoreto CAO. Revisão de prontuário como estratégia de ensino-aprendizagem da medicina centrada na pessoa em um ambulatório universitário no município do Rio de Janeiro. Rev Bras Med Fam Comunidade. 2014; 9(30):96-103.

8. Brasil. Ministério da Educação. Conselho Nacional de Educação, Câmara de Educação Superior. Resolução CNE/CES n% 4 de 7 de novembro de 2001. Institui diretrizes curriculares nacionais do curso de graduação em Medicina. Diário Oficial da União. Brasília, 9 nov. 2001; Seção 1, p.38.

9. Santos ML, Novaes CO, Iglesias AC. Epidemiological profile of patients seen in the pre-anesthetic assessment clinic of a university hospital. Rev Bras Anestesiol. 2017; 67(5): 457-467.

10. Guedes BAP, Vale FLB, Souza RW, Costa MKA, Batista SR. A Organização Da Atenção Ambulatorial Secundária na SESDF. Ciênc. saúde colet. 2019; 24(6):2125-2134.

11. Vasconcelos GMT, Santos GKBB, Santos ACFS, Silva JOM, Santos ES. Perfil sociodemográfico dos pacientes atendidos em um ambulatório público de cuidados com feridas complexas. Anais Congresso Internacional de Enfermagem; 2019; Sergipe, Unit, 2019.

12. Pexe M, Carvalho DLBC, Menotti AFS, Soares JGS, Jardim NA, Casalenuovo PRM. Perfil epidemiológico do Ambulatório de Saúde Mental Infantojuvenil da Clínica Integrada do Centro Universitário de Várzea Grande (UNIVAG). Caderno de Publicações Univag. 2019; 10:4-14.

13. Adamczyk SP, Souza SJP. Perfil epidemiológico dos pacientes atendidos no ambulatório de enfermagem. Rev Gestão e Saúde. 2018; 19(1): 118-28.

14. Dantas MM, Solá ALN, Pires DAL, Silva JBP, Reis MAC, Herrera MR, Herrera SDSC. Profile of Patients Attended in the Cardiology
Ambulatory of the South Region of Tocantins in the Year 2015. Revista Cereus. 2019; 11(2):1-11.

15. Hodniki PP, Teixeira CRS, Zanetti ML, Moraes C, Fermino RC, Berra S. Prática de atividade física e ambiente percebido de usuários do Sistema Único de Saúde. Saúde coletiva. 2021;11(65): 6280-87.

16. Brasil. Pesquisa Nacional de Saúde: 2013: acesso e utilização dos serviços de saúde, acidentes e violências: Brasil, grandes regiões e unidades da federação / IBGE, Coordenação de Trabalho e Rendimento - Rio de Janeiro: IBGE, 2015.

17. Sena NV, Alves AFV, Pontes LM, Silva PRS, Duffles MSL, Souza MLP, Brandão BJF. Perfil epidemiológico dos pacientes atendidos no serviço de dermatologia da BWS. BWS Journal. 2020; 3:1-9.

18. Saraiva LGF, Dornelas PG, Cau SBA, Calábria LK. Perfil epidemiológico de pacientes atendidos em uma rede ambulatorial do Hiperdia Minas em Governador Valadares-MG. Revista de Atenção à Saúde. 2016; 14(48): 40-47.

19. Viacava F, Porto SM, Carvalho CC, Bellido JG. Desigualdades regionais e sociais em saúde segundo inquéritos domiciliares (Brasil, 1998-2013). Ciênc. saúde colet. 2019; 24(7): 2745-60.

20. Franco RJS. A atividade física no presente pode ser a receita para evitar os males da obesidade e hipertensão no futuro. Arq. Bras. Cardiol. 2020; 15(1): 50-51.

21. Lemos LN, Tavares RMF, Donadelli CAM. Perfil epidemiológico de pacientes com colelitíase atendidos em um ambulatório de cirurgia. Revista Eletrônica Acervo Saúde/ Electronic Journal Collection Health. 2019;28(28):1-9.

22. Silva LCC, Araújo AJ, Queiroz AMD, Sales MPU, Castellano MVCO. Controle do tabagismo: desafios e conquistas. J. bras. pneumol. 2016; 42(2): 290-298.

23. Brasil. Ministério do Trabalho e Emprego - MTE. Relação Anual de Informações Sociais - RAIS, 2013. Disponível em <http://www. mte.gov.br/rais/default.asp>. Acesso em: 28 jul. 2020.

24. Gouveia N, Leon AP, Junger W, Lins JF, Freitas CU de. Poluição do ar e impactos na saúde na Região Metropolitana de Belo Horizonte - Minas Gerais, Brasil. Ciênc. saúde colet. 2019; 24(10): 3773-81.

25. Lemos AP, Ribeiro C, Fernandes J, Bernardes K, Fernandes R. Saúde do homem: Os motivos da procura dos homens pelos serviços de saúde. Rev. enferm. UFPE online. 2017; 11(11): 4546-53.

26. Sousa RJ, Soares TM, Rosario CR, Rosa DPS, David RAR, Brito HES. Aspectos da masculinidade como impeditivo do autocuidado na saúde do homem. Saúde coletiva. 2021; 11(65): 6306-6314.

27. Soares MOM, Higa EFR, Passos AHR, Ikuno MRM, Bonifácio LA, Mestieri CP, Ismael RK. Reflexões contemporâneas sobre anamnese na visão do estudante de Medicina. Rev. bras. educ. med. 2014; 38(3): 314-22. 